\title{
Transforming Nursing Practice through Digitalization
}

Happy Nursing Week! In this special issue of the Canadian Journal of Nursing Leadership for Nursing Week, you will find much food for thought. This issue may surprise you while you ponder the workings of Humber River Hospital, the first fully digitalized hospital in North America. Dr. Vanessa Burkoski and her team of researchers present their findings and insights related to the various technologies and the ways in which they impact nurses' work and patients' experiences of care. I imagine you will read with anticipation the ways in which digital health care practices have transformed nursing practice and the patient experience at Humber River Hospital.

When asked if I would agree to be guest editor, I shared with the editorial director some of my thoughts about digitalization. I'll pose a few here as I am wondering if, as you read this special issue, you might like to share some of yours with the authors.

First, what interested me was the effect of these technologies on the elderly and frail elderly, as well as those who are not technologically savvy. I was concerned that my own father, who remained a staunch Luddite by choice, might not have ever wished to learn about new technology such as bedside terminals when in hospital. He had the gift of conversation and humour and loved talking to the nurses, physicians and other patients. In the papers on patient empowerment and smartphone technology, the authors address the potential challenges for the elderly from a nursing perspective. Although the authors see this as a challenge, they find that the challenge is balanced by the opportunities and the many benefits that are achieved in the digitalized hospital, such as patient safety, time management and the potential for patient empowerment.

Other thoughts and questions come to mind. What if the technology fails? What effect might this, or the potential for less than effective use of technology, have on patient care? In her introductory piece on nursing leadership in the fully digital practice realm, Dr. Burkoski notes that nurse leaders will have to intervene to maximize the effective use of technology and that through their understanding of the principles of nursing informatics, quality assurance, etc., they will lay the foundation for engaging staff. 
As well, I thought about the nurses. How much of their time was invested in learning the technology? Did they need special training? Does this digitalized hospital attract nurses or cause them to go elsewhere? In the paper on optimizing nursing practice, the authors discuss in depth the kinds of support that nurses are offered. RNAO's Best Practice Guidelines (BPGs), for example, have been embedded in the electronic medical record where the BPGs will serve to supplement the knowledge that nurses already bring to their work. The paper on the experiences of nurses working in a fully digitalized hospital uncovers nurses' perception of technology, whereas the paper on generational differences addresses training for the technological competence needs of nurses across generations.

Another thought: does each form of technology contribute to better healthcare? The What We Learned section in the paper on the closed-loop medication (CLM) system highlights the effectiveness of CLM technology, whereas the paper on violence prevention shares the finding that engaging staff is critical for successful implementation, evaluation and modification of technology.

I also wondered what is the "right" or "best" ratio of technology to human staff? I thought about the work of Donna Haraway (1991), a self-identified cyborg feminist and historian of science who has written about cyborgs and humans. She defines a cyborg as a "hybrid creature composed of organism and machine" (p. XI). In her book entitled Simians, Cyborgs and Women: The Reinvention of Nature, Haraway (1991) makes the following claim:

High tech culture challenges ... dualisms in intriguing ways. It is not clear who makes and who is made in the relation between human and machine (p. 177).

As I reread Haraway's work, I wondered if digitalization has caused or will cause nurses to become so attached to the technology that they appear to some as cyborgs, with the technology becoming an extension of their bodies. Does it have an effect on their ability to give care? Who is in charge of care decisions: nurse or machine?

The article on patient empowerment notes that the technology allows patients to have greater authority over their own health information. This is long overdue. But will this contribute to or spark power struggles over who has the right to know? I wonder whether patients will have the right to deny certain health professionals access to their health information. Will they be able to, in effect, choose who can view their records?

As you read each article, your questions may be answered or you may find that certain papers raise more questions than they answer. You may find aspects of the digitalization of healthcare, as explored in this issue, exciting and may hunger for more 
information, or you may disagree with certain aspects. All perspectives are critical to the ongoing dialogue around digital practice. This issue is meant to spark conversation, and you are invited to contact the authors with your questions, concerns, or reactions.

I am left with wondering what will come next in the digital healthcare world. Certain questions remain, such as what care is appropriate to be delivered or supported by digitalized forms of technology and what care should remain in the hands of the nurse? Which forms of technology contribute to better healthcare? In a number of papers in this issue, the authors have worked with questions such as these. They also clearly note that these are areas to follow up and address.

This special issue is exciting not only for the insights it offers us as nurses and nurse leaders but also, more notably, the many questions the papers will generate. In 2019, we must certainly begin to attend to them.

Sandra DeLuca RN, PhD

Associate Dean, School of Nursing

Fanshawe College

Adjunct Associate Professor

Arthur Labatt Family School of Nursing

Western University

London, $\mathrm{ON}$

\section{References}

Haraway, D.J. 1991. Simians, Cyborgs and Women: The Reinvention of Nature. New York: Routledge. 


\section{$\mathbf{H}_{\substack{\text { Hosmegra neve } \\ \text { Hosith }}}^{+}$}

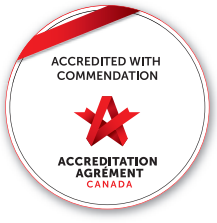

DOO
$\mathbf{D}$ BEST PRACTICE
SPOTLIGHT
ORGANIZATION
C A N A D A A
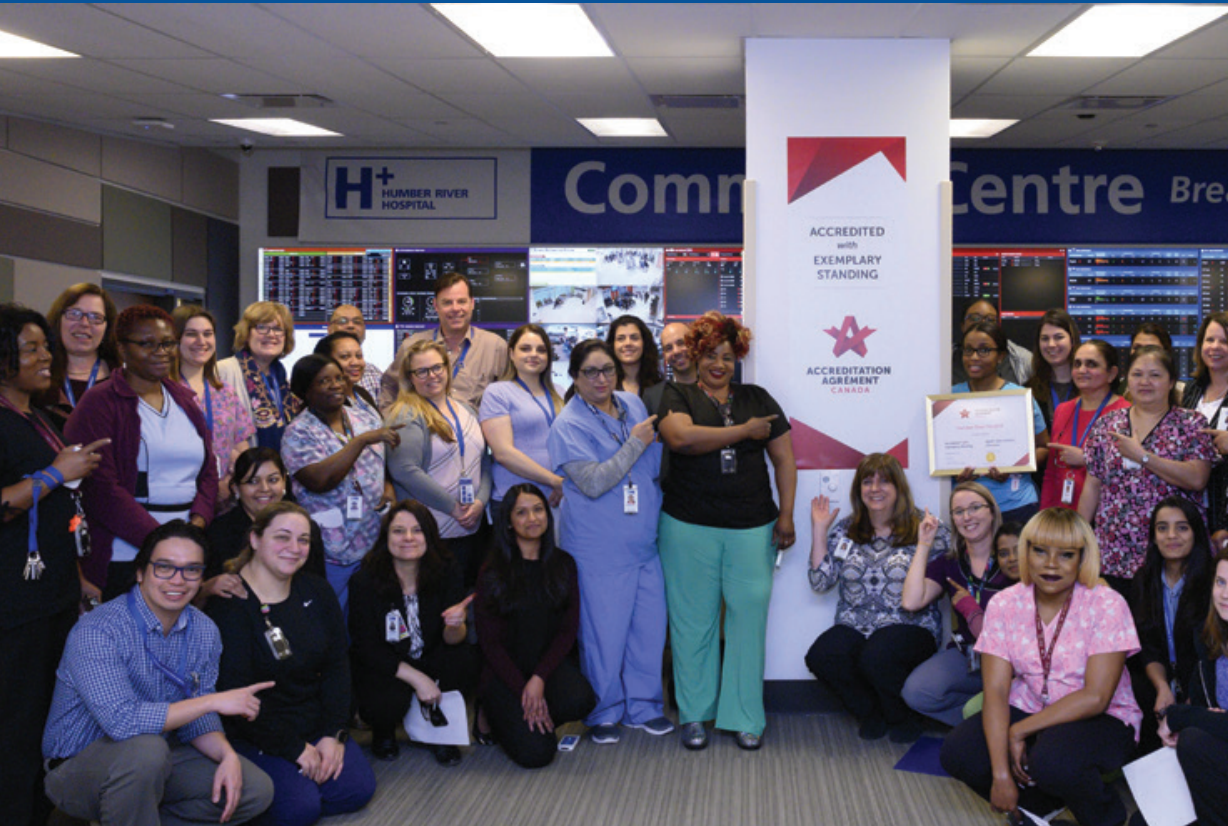

ACCREDITED

entre Breakthrough Intelligence

STEMPLARY

* cactorernon
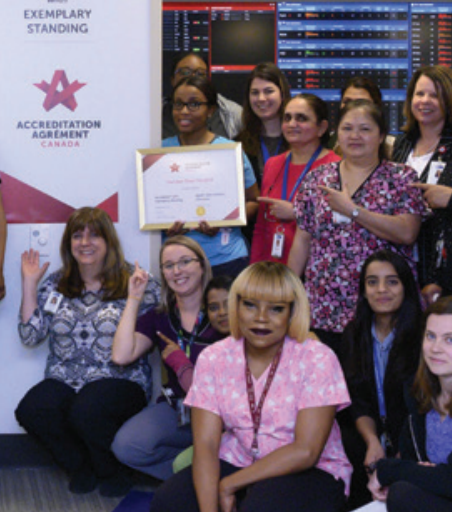

$\sin 2$
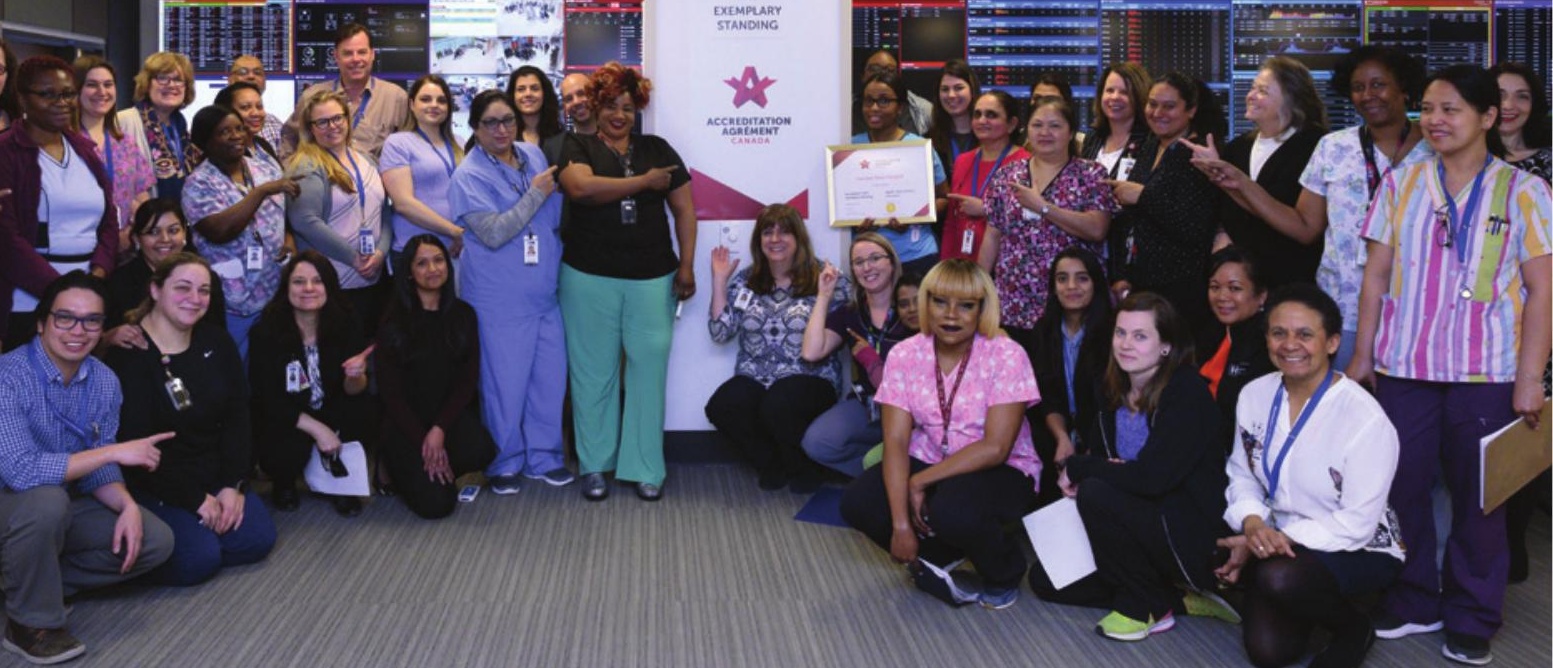

\section{Leading technology \& innovation integration in nursing}




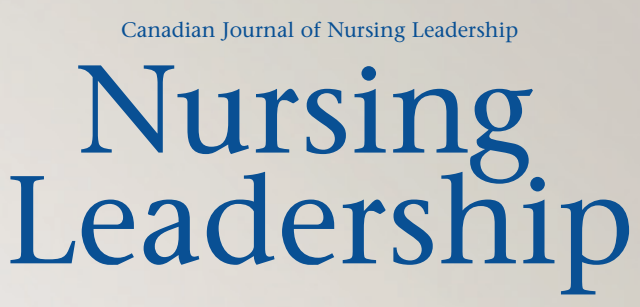

Leadership in Nursing Management, Practice, Education \& Research

NURSING LEADERSHIP - PERSPECTIVES AND INSIGHTS

Building Healthcare Leadership Capacity: Strategy, Insights and Reflections 10

The Role of Education in Developing Leadership in Nurses

Engaging Nurses in Future Management Careers: Perspectives on Leadership and Management Competency Development through an Internship Initiative 36

Black Nurse Leaders in the Canadian Healthcare System 50

Politics • Policy • Theory • Innovation

Us

ACEN
ACDSI

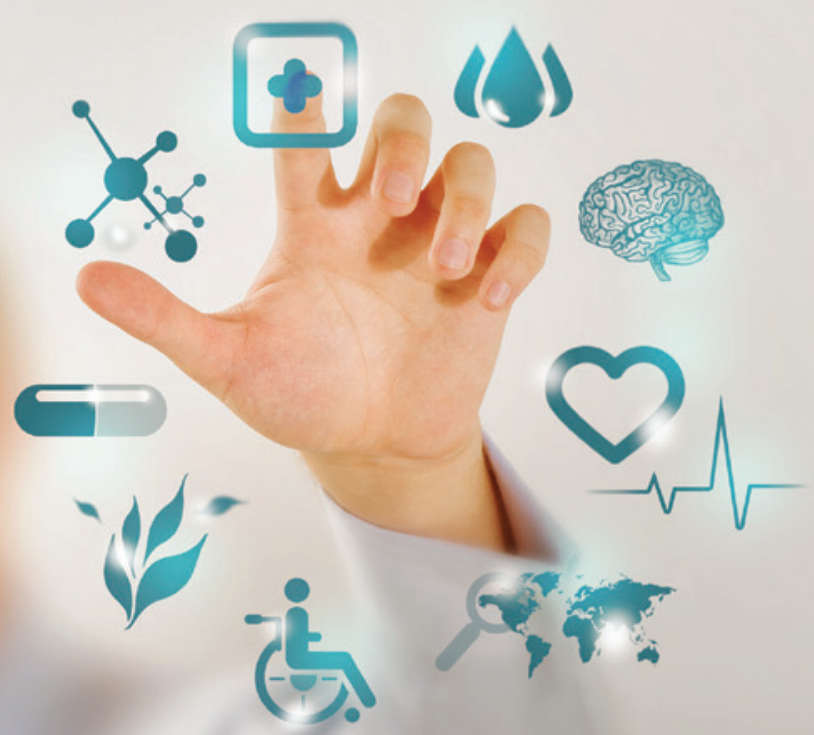

Covering politics, policy, theory and innovations that contribute to leadership in nursing administration, practice, teaching and research. 